Cahiers
de a Recherche
Fon les Droits
Fondamentaux

Cahiers de la recherche sur les droits fondamentaux

$18 \mid 2020$

La vulnérabilité

\title{
La vulnérabilité en droit privé
}

\section{Laurence Fin-Langer}

\section{OpenEdition}

Journals

Édition électronique

URL : https://journals.openedition.org/crdf/6402

DOI : $10.4000 /$ crdf.6402

ISSN : 2264-1246

Éditeur

Presses universitaires de Caen

Édition imprimée

Date de publication : 19 novembre 2020

Pagination : 11-19

ISBN : 978-2-84133-987-7

ISSN : 1634-8842

Référence électronique

Laurence Fin-Langer, «La vulnérabilité en droit privé », Cahiers de la recherche sur les droits

fondamentaux [En ligne], 18 | 2020, mis en ligne le 19 novembre 2021, consulté le 14 novembre 2022

URL : http://journals.openedition.org/crdf/6402 ; DOI : https://doi.org/10.4000/crdf.6402 


\title{
La vulnérabilité en droit privé
}

\author{
Laurence FIN-LANGER \\ Professeure de droit privé à l'université de Caen Normandie \\ Institut Demolombe (EA 967)
}

I. La reconnaissance des vulnérabilités par le droit privé

A. Les vulnérabilités reconnues

1. Des vulnérabilités implicitement reconnues

2. Des vulnérabilités explicitement reconnues

B. Des vulnérabilités discutées

1. Une liste des personnes vulnérables discutée

2. Une liste de facteurs de vulnérabilité discutée

\section{Le traitement des vulnérabilités par le droit privé}

A. L'insuffisance des mesures n'assurant pas une prise en charge de la personne vulnérable

1. L'insuffisance de la sanction de l'exploitation de la vulnérabilité

2. L'insuffisance de la protection de la personne vulnérable par des dispositions non spécifiques

B. La mise en place nécessaire de mesures assurant la prise en charge de la personne vulnérable

1. La prise en charge individuelle de la personne vulnérable

2. La prise en charge collective de la personne vulnérable

Dans le langage courant, la vulnérabilité peut être définie comme le caractère de quelqu'un qui peut être blessé, atteint physiquement, évoquant l'idée de fragilité et de faiblesse. Ce concept, transversal, a fait l'objet d'études aussi bien en sociologie, qu'en économie, en philosophie ${ }^{2}$, en santé ${ }^{3}$, comme en témoignent plusieurs colloques

\begin{abstract}
Aimons ce mot de vulnérabilité, voyons-le vivre ou faisons-le vivre selon nos places respectives. Il est la passerelle entre l'œuvre législative et la protection réelle des majeurs les plus fragiles. Légiférer sur la vulnérabilité est possible et même nécessaire [... $]^{1}$.
\end{abstract}

1. T. Fossier, «Peut-on légiférer sur la vulnérabilité?», Droit de la famille, nº 2, 2011, dossier 2.

2. Voir par exemple M. Garrau, L'importance de la vulnérabilité: essai sur la signification et les implications de la catégorie de vulnérabilité dans la philosophie morale et politique contemporaine, thèse de doctorat en philosophie, université Paris 10, 2011; M. Garrau, Politiques de la vulnérabilité, Paris, CNRS éditions, 2018; Y. Pillant, Une politique de la vulnérabilité est-elle "pensable»?, thèse de doctorat en philosophie, université d'Aix-Marseille, 2018.

3. Voir, pour une présentation des différentes conceptions aussi bien en France qu’à l'étranger: H. Thomas, "Vulnérabilité, fragilité, précarité, résilience, etc. De l'usage et de la traduction de notions éponges en sciences de l'homme et de la vie», Recueil Alexandries (Esquisses), 2008, en ligne: https://www.reseau-terra.eu/article697.html.

4. La vulnérabilité: questions de recherche en sciences sociales (Actes du colloque tenu à l'université Versailles-Saint-Quentin-en-Yvelines, 29 avril 2011), M. Bresson, V. Geromini, N. Pottier (dir.), Fribourg, Academic Press Fribourg (Res Socialis), 2013 ; « Penser/ exposer la vulnérabilité», colloque dirigé par N. Proia-Lelouey, M.-H. Boblet, H. Marche, université de Caen Normandie, 29 novembre-1 ${ }^{\text {er }}$ décembre 2018. 
qui, ici, est celui de devenir pauvre ${ }^{5}$. Le droit privé s'est également emparé de cette notion. C'est le droit pénal qui a introduit cette notion en 1980 pour aggraver la répression du viol et de certains attentats aux mœurs. Depuis, le Code pénal est celui qui contient le nombre d'occurrences le plus élevé ${ }^{6}$. Le droit civil a également été imprégné de cette notion, que ce soit en droit des contrats ${ }^{7}$ ou en droit des personnes protégées ${ }^{8}$, bien que le Code civil ne mentionne pas expressément ce terme. On peut également citer le Code de la consommation, le Code de procédure pénale, le Code de l'urbanisme, le Code de l'entrée et du séjour des étrangers et du droit d'asile ${ }^{9}$. Plusieurs études transversales, dont le rapport annuel de la Cour de cassation publié en $2009^{10}$, ont été menées ${ }^{11}$. Malgré sa diffusion et sa reconnaissance en tant que véritable notion ${ }^{12}$, ce concept est contesté en raison de son caractère flou et peu opérationnel ${ }^{13}$. Ainsi, il n'est pas cité dans plusieurs dictionnaires juridiques ${ }^{14}$. Pourtant derrière cette notion se cachent des piliers fondamentaux du droit privé comme la personne, sa dignité et la justice sociale. Quelles sont donc les vulnérabilités reconnues et à reconnaître en droit privé? Comment sont-elles ou doivent-elles être traitées? Cette étude tentera d'apporter des pistes de réflexion.

\section{La reconnaissance des vulnérabilités par le droit privé}

Le droit en reconnaît plusieurs formes, mais se pose la question d'une éventuelle extension.

\section{A. Les vulnérabilités reconnues}

Certaines vulnérabilités sont nommées alors que d'autres sont innommées ou implicites.

\section{Des vulnérabilités implicitement reconnues}

Certaines branches du droit protègent des personnes présumées vulnérables, non pas en tant que telles mais en raison d'un rapport de force et donc de manière relative : elles sont présumées être la partie faible d'une relation. Il en est ainsi par exemple du consommateur ou du salarié. Vulnérabilité est alors synonyme d'infériorité, forme de faiblesse extrinsèque, contingente par comparaison à une autre partie considérée comme forte. Cette infériorité peut résulter d'une dépendance économique, informationnelle ou juridique, altérant l'intégrité ou la liberté du consentement de ces personnes présumées vulnérables. Le droit assure alors leur protection, sans pour autant utiliser l'expression de vulnérabilité. Si elle existe, elle n'est pas nécessairement mise en avant. La vulnérabilité peut aussi être synonyme d'une faiblesse inhérente à la personne. Le droit des personnes permet ainsi de protéger et d'accompagner les mineurs et certains majeurs en raison d'une vulnérabilité personnelle liée à leur âge, à leur faiblesse physique, à leur santé les empêchant d'exercer leurs droits. Ils sont alors vulnérables indépendamment de leur partenaire ${ }^{15}$. Il en est de même en droit pénal, voire en droit de la santé, qui considèrent le mineur de 15 ans de manière irréfragable comme vulnérable en raison de sa faiblesse physique qui l'empêche de se défendre.

Cette reconnaissance implicite et présumée soulève plusieurs interrogations, comme le choix du seuil retenu. Pourquoi le critère relatif aux mineurs n'est pas toujours et n'a pas toujours été de 15 ans? Cette présomption repose sur un postulat parfois contraire à la réalité et arbitraire. Un adolescent de 16 ans peut être vulnérable et un autre ne le sera plus nécessairement dès 14 ans. Il en est de même des vulnérabilités résultant de l'infériorité présumée. Cela a aussi pour risque de créer des surprotections et des

5. J. É. Bidou, I. Droy, «Peut-on mesurer la vulnérabilité sociale et économique des ménages et des individus?», communication au colloque «La mesure du développement», Paris, février 2012.

6. Au 15 janvier 2020, une recherche sur le site Légifrance donne trente-deux références dans le Code pénal.

7. N. Pfalzgraf, Vulnérabilité et vices du consentement, thèse de doctorat en droit privé, université de Strasbourg, 2015; S. Le Gac-Pech, «Bâtir un droit des contractants vulnérables", Revue trimestrielle de droit civil, 2014, p. 581.

8. J. Hauser, «Des incapables aux personnes vulnérables», Droit de la famille, $\mathrm{n}^{\circ} 5,2007$, étude 14 ; «Les personnes vulnérables », $102^{\mathrm{e}}$ congrès des notaires de France, Strasbourg, mai 2006.

9. Une recherche sur Légifrance, au 31 janvier 2020, donne vingt-deux codes dans lesquels le mot apparaît.

10. X. Lagarde, "La protection des personnes vulnérables, entre audace et tempérance. À propos du rapport annuel 2009 de la Cour de cassation», La semaine juridique, édition générale, $\mathrm{n}^{\circ}$ 17, 26 avril 2010, 463; Cour de cassation, Les personnes vulnérables dans la jurisprudence de la Cour de cassation, rapport annuel de la Cour de cassation 2009, Paris, La documentation française, 2009, en ligne: https://www.courdecassation.fr/ publications_26/rapport_annuel_36/rapport_2009_3408.

11. V. Gittard, Protection de la personne et catégories juridiques: vers un nouveau concept de vulnérabilité, thèse de doctorat en droit privé, université Paris 2, 2005; L. Dutheil-Warolin, La notion de vulnérabilité de la personne physique en droit privé, thèse de doctorat en droit, université de Limoges, 2004; F.-X. Roux-Demare, «La notion de vulnérabilité, approche juridique d'un concept polymorphe », Les cahiers de la justice, n 4 , 2019, p. 619-630; M. Blondel, La personne vulnérable en droit international, thèse de doctorat en droit public, université de Bordeaux, 2015, en ligne: https://tel.archives-ouvertes.fr/tel-01424139/document; C. Ruet, La vulnérabilité dans la jurisprudence de la Cour européenne des droits de l'homme, en ligne: https://hal-univ-paris1o.archives-ouvertes.fr/hal-o1671495/document.

12. L. Dutheil-Warolin, La notion de vulnérabilité...

13. F. Terré, D. Fenouillet, Droit civil: les personnes, $8^{\mathrm{e}}$ éd., Paris, Dalloz (Précis. Droit privé), 2012, nº 314 ; P. Malaurie, L. Aynès, Droit des personnes: la protection des mineurs et des majeurs, $10^{\mathrm{e}}$ éd., Paris, LGDJ, 2018, $\mathrm{n}^{\circ} 492$.

14. Il n'est défini ni dans le Lexique des termes juridiques 2019-2020, $27^{\mathrm{e}}$ éd., S. Guinchard, T. Debard (dir.), Paris, Dalloz, 2019, ni dans le Dictionnaire $d u$ vocabulaire juridique 2017, $8^{\mathrm{e}}$ éd., R. Cabrillac (dir.), Paris, LexisNexis, 2016, ni dans le Dictionnaire de la culture juridique, D. Alland, S. Rials (dir.), Paris, Lamy - Presses universitaires de France (Quadrige), 2003.

15. La doctrine majoritaire parle du droit des personnes vulnérables: A. Batteur, Droit des personnes, des familles et des majeurs protégés, 10 éd., Issyles-Moulineaux, LGDJ, 2019, n 1268 sq.; N. Peterka, F. Arbellot, A. Caron-Déglise, Protection de la personne vulnérable, $4^{\mathrm{e}}$ éd., Paris, Dalloz (Dalloz 
effets pervers ${ }^{16}$ : la protection ne va pas nécessairement cibler la personne réellement vulnérable. Pour répondre à ces critiques, le droit a donc reconnu des vulnérabilités explicites, parfois qualifiées de particulières.

\section{Des vulnérabilités explicitement reconnues}

Le droit pénal retient depuis 1980 des causes explicites de vulnérabilité: la minorité, l'état de grossesse, la maladie, une infirmité ou une déficience physique ou mentale. Il s'agit, dans ces cas, d'une vulnérabilité personnelle liée à la personne ${ }^{17}$ révélant une faiblesse physique mais aussi mentale ${ }^{18}$. Dans des textes plus récents ${ }^{19}$, apparaît également la vulnérabilité en raison d'une situation économique ou sociale, la personne étant isolée. Il s'agit alors d'une vulnérabilité réelle ou de circonstance. D'autres codes font également référence à cette vulnérabilité économique ${ }^{20}$ et sociale ${ }^{21}$. La Cour européenne des droits de l'homme désigne quant à elle des groupes de population considérés comme vulnérables, ces personnes ayant été victimes, dans le passé, de traitements défavorables, voire dégradants, aux conséquences durables ${ }^{22}$. Le rapport de la Cour de cassation met enfin l'accent sur la vulnérabilité juridique, pour les personnes étrangères faisant l'objet d'une privation de liberté sur décision administrative. Est-il possible d'en admettre d'autres?

\section{B. Des vulnérabilités discutées}

Cette notion de vulnérabilité joue un rôle juridique précis : si les droits sont reconnus, ils doivent aussi être effectifs tel qu'il en ressort de la jurisprudence de la Cour européenne des droits de l'homme ${ }^{23}$, et la vulnérabilité permet ce passage ${ }^{24}$, car elle est "plus attentive à la personne incarnée qu'au sujet abstrait de droit ${ }^{25}$. Elle offre un moyen de corriger une situation dans laquelle la personne ne peut effectivement exercer un droit en raison d'une faiblesse particulière. Si le droit a reconnu un certain nombre de situations, d'autres peuvent également être prises en compte.

\section{Une liste des personnes vulnérables discutée}

La vulnérabilité est souvent réservée aux seules personnes physiques. Faut-il aller au-delà et si oui à qui ou à quoi étendre cette notion? La littérature juridique fait déjà référence à la vulnérabilité des milieux ${ }^{26}$, des biens ${ }^{27}$, des édifices ${ }^{28}$ ou des animaux ${ }^{29}$. Le Fonds monétaire international (FMI) a également établi des indicateurs de vulnérabilité des États, au regard notamment de leur capacité financière, afin de préconiser des mesures pour éviter l'apparition d'une crise financière qui serait dommageable pour l'ensemble de la population ${ }^{30}$. La question n'est donc pas saugrenue. L'approche qui vise à protéger la personne privée de l'exercice de ses droits en raison d'un risque particulier conduit à étendre cette vulnérabilité à toutes les personnes juridiques au nom d'une égalité de traitement. La personne est alors définie de manière abstraite comme étant la possibilité d'être un acteur juridique et d'avoir des droits et des obligations. Pourraient ainsi être d'ores et déjà concernées les personnes morales ${ }^{31}$, au nom d'un «anthropomorphisme $»^{32}$ ou d'un «technopersonnalisme ${ }^{33}$, déjà présent en droit interne

action), 2017; A. Marais, Droit des personnes, $3^{\mathrm{e}}$ éd., Paris, Dalloz (Cours Dalloz), 2018, $\mathrm{n}^{\circ} 323$ sq.; Protection juridique des majeurs vulnérables, rapport du défenseur des droits, septembre 2016. En revanche, d'autres préfèrent le vocable d'incapables: B. Teyssié, Droit des personnes, $19^{\mathrm{e}}$ éd., Paris, LexisNexis, 2017, n 561; P. Malaurie, L. Aynès, Droit des personnes..., nº 492; P. Courbe, F. Jault-Seseke, Droit des personnes, de la famille et des incapacités, Paris, Dalloz (Mémentos Dalloz), 2018, p. 217 sq.

16. Voir, dans ce même numéro, la contribution de D. Bazin-Beust et J.-J. Thouroude, «La vulnérabilité des usagers du service public et des consommateurs".

17. Distinction proposée par X. Lagarde, avant-propos au rapport annuel de la Cour de cassation, Les personnes vulnérables..., p. 64.

18. Le terme "vulnérabilité» est défini uniquement dans cette conception personnelle dans le Vocabulaire juridique de G. Cornu, 10 éd., Paris, Presses universitaires de France, 2014, ou dans le Dictionnaire juridique de C. Puigelier, $2^{\mathrm{e}}$ éd., Bruxelles, Bruylant (Paradigme), 2017.

19. Code pénal, art. 222-24 et 222-33.

20. Code du travail, art. L. 1132-1; Code de l'action sociale et des familles, art. L. 266-1; Code des transports, art. L. 1231-1-1 et L. 1215-3.

21. Code général des collectivités locales, art. L. 2333-87; Code de l'action sociale et des familles, art. L. 266-1; Code des transports, art. L. 1231-1-1, L. 1215-3 et L. 1215-4.

22. C. Ruet, La vulnérabilité dans la jurisprudence..., p. 9.

23. Voir, par exemple, la mise en place d'un système d'aide juridictionnelle pour garantir un accès effectif à un juge (Cour EDH, 9 octobre 1979, Airey c. Irlande, $\mathrm{n}^{\circ} 6289 / 73, \$ 26$ ), sauf en raison des circonstances de l'affaire, notamment la gravité de l'enjeu pour le requérant (Cour EDH, 15 février 2005, Steel et Morris c. Royaume-Uni, n 68416/01, \$61).

24. X. Lagarde, avant-propos au rapport annuel de la Cour de cassation, Les personnes vulnérables..., p. 56; D. Guérin, «La notion de vulnérabilité appliquée à l'animal? ", in L'animal et l'homme, F.-X. Roux-Demare (dir.), Paris, Mare \& Martin, 2019, p. 52.

25. F. Terré, D. Fenouillet, Droit civil: les personnes, $\mathrm{n}^{\circ} 314$.

26. Par exemple: Code de l'environnement, art. R. 556-2; Code de l'urbanisme, art. L. 122-15 (vise les espaces montagnards); Code de l'environnement, art. R. 222-2 (cite les régions); Code de l'environnement, art. L. 566-7 et R. 229-51 (mentionne les territoires).

27. Par exemple: Code de l'environnement, art. L. 561-3.

28. Code de la construction et de l'habitat, art. L. 124-1; Code de la défense, art. L. 1322-3.

29. D. Guérin, «La notion de vulnérabilité appliquée à l’animal?», p. $47 \mathrm{sq}$.

30. Voir la fiche technique du FMI, «Indicateurs de vulnérabilité», en ligne: https:/www.imf.org/external/np/exr/facts/fre/vulf.htm.

31. Voir, dans ce même numéro, le rapport d'A. Bennini, «Vulnérabilité et sociétés».

32. Voir V. Wester-Ouisse, «Dérives anthropomorphiques de la personnalité morale: ascendances et influences», La semaine juridique, édition générale, $\mathrm{n}^{\mathrm{o}}$ 16-17, 2009, doctr. 137; J. Rochfeld, Les grandes notions du droit privé, $2^{\mathrm{e}}$ éd., Paris, Presses universitaires de France (Thémis), 2013, p. 87: mouvement qui attribue les mêmes droits et obligations aux personnes morales que ceux dont dispose la personne physique.

33. G. Loiseau, «Des droits humains pour personnes non humaines», Recueil Dalloz, 2011, p. 2558. 
ou supranational ${ }^{34}$. D'autres, à l'avenir, pourraient aussi devenir des personnes au sens juridique et technique, comme les robots dotés d'une intelligence artificielle ${ }^{35}$, les animaux ${ }^{36}$, voire la nature ${ }^{37}$. Cette conception présente plusieurs risques. Le premier est d'étendre tellement la notion de vulnérabilité qu'elle perdrait tout intérêt. Le deuxième est de mettre sur un pied d'égalité des sujets de droit qui ne sont cependant pas comparables. Si leurs intérêts doivent aussi être protégés, cette protection ne peut être identique à celle accordée aux personnes humaines, au risque de diluer l'être humain au milieu des sujets de droit et de voir disparaître sa primauté dans l'ordre juridique, primauté nécessaire car justifiée par son humanité ${ }^{8}$. Pour éviter cette dérive, il pourrait être envisagé de parler, pour ces autres sujets titulaires de certains droits voire d'obligations ${ }^{39}$, de "parapersonnalité ».

Mais la personne n'est pas qu'une catégorie juridique spécifique, permettant d'accéder à la vie juridique. Une conception concrète est nécessaire: la personne est aussi un être humain doté d'un corps et d'un esprit, appartenant à l'espèce humaine ${ }^{40}$. À ce titre, la personne dite «humaine» doit être protégée non seulement dans l'exercice de ses droits mais aussi dans sa dignité ${ }^{41}$, c'est-à-dire en réalité dans son humanité au-delà de la personne ${ }^{42}$. La vulnérabilité peut ainsi être définie plus restrictivement comme étant «une atteinte à la personnalité juridique, [...] fai[san]t peser une menace sur la dignité des individus ${ }^{43}$. C'est donc la dignité de l'être humain qui fonde le respect dû à la personne humaine ${ }^{44}$. Elle peut être vue comme un principe matriciel et le fondement des droits de l'homme ${ }^{45}$. Cette approche limite ainsi la portée de la vulnérabilité à la seule personne physique, quelle que soit sa qualité: elle peut agir en tant que salarié, consommateur, cocontractant ou travailleur indépendant ${ }^{46}$, etc. Ce qui compte c'est la personne physique qui agit et cela dans toutes ses dimensions et identités. Il convient alors de la protéger dès lors qu'en raison de sa faiblesse ou de sa fragilité elle ne peut faire face à une situation qui l'expose à un risque particulier. Mais quel risque?

\section{Une liste de facteurs de vulnérabilité discutée}

Le rapport de la Cour de cassation de 2009 fait une liste très complète d'hypothèses:

[...] les enfants, les étrangers placés en zone de rétention, les majeurs protégés, les emprunteurs adhérents à une assurance de groupe, toutes les personnes prises en qualité d'assurés sociaux, les bénéficiaires de l'aide juridictionnelle, les particuliers surendettés, partie des locataires de leur logement principal, les investisseurs, les emprunteurs et les cautions profanes, les professionnels en situation de dépendance économique, les personnes âgées ou celles dont la santé est altérée, les salariés précaires, les salariés protégés, les stagiaires, les personnes physiquement et psychologiquement faibles, les $"$ nouveaux esclaves ${ }^{47}$.

Derrière cette énumération à la Prévert, on décèle souvent les mêmes facteurs de vulnérabilité. Certes, c'est sans doute le propre de l'être humain que de prendre des risques dès lors qu'il agit. Ce n'est donc pas n'importe quelle exposition à un risque quelconque ${ }^{48}$. Il semble effectivement que le simple fait d'être salarié ou consommateur ne suffise pas pour être considéré comme vulnérable, même s'il s'agit de la partie considérée comme faible dans la relation contractuelle. Le législateur conscient de cette conception trop large évite précisément ce vocabulaire et va utiliser ensuite "particulière vulnérabilité » ${ }^{49}$, qui est sans doute la seule. Ainsi, le salarié pourrait se trouver dans une situation de vulnérabilité en raison notamment de sa situation personnelle mais aussi des conditions de travail dans lesquelles il effectue sa prestation. Plusieurs critères

34. J. Rochfeld, Les grandes notions du droit privé, p. 89 sq.; p. 126 sq. : octroi aux personnes morales de certains droits fondamentaux comme le droit au respect des biens, la liberté d'expression et même la liberté religieuse et les droits de la personnalité.

35. A. Bensoussan, «La personne robot», Recueil Dalloz, 2017, p. 2044; X. Labbée, «Faut-il personnifier la voiture autonome?», Recueil Dalloz, 2019, p. 1719; P. Malaurie, L. Aynès, Droit des personnes..., $\mathrm{n}^{\circ}$ 2. Pour une illustration possible d'une telle évolution, voir la série suédoise Real Humans, diffusée par Arte entre 2012 et 2014.

36. Pour l'instant, le législateur ne les a pas reconnus comme telles mais comme «des êtres vivants doués de sensibilité» (Code civil, art. 515-14). Mais certains auteurs sont pour la reconnaissance d'une personnalité dite technique, à l'instar de ce qui existe pour les personnes morales, pour renforcer leur protection: J.-P. Marguénaud, F. Burgat, J. Leroy, «La personnalité animale», Recueil Dalloz, 2020, p. 28.

37. M. Hautereau-Boutonnet, «Faut-il accorder la personnalité juridique à la nature?», Recueil Dalloz, 2017, p. 1040.

38. G. Loiseau, «Des droits humains...».

39. Les personnes morales ont une personnalité juridique dont les droits et les obligations devraient dépendre de la finalité spécifique. C'est d'ailleurs la même approche soutenue par Jean-Pierre Marguénaud, Florence Burgat et Jacques Leroy pour fonder la cause d'une personnalité animale technique sans la calquer intégralement sur la personne physique (J.-P. Marguénaud, F. Burgat, J. Leroy, "La personnalité animale»). Le risque d'utiliser le terme de personne en droit est d'avoir un alignement des droits pour tous les textes qui ne précisent pas la nature de la personne concernée.

40. J. Rochfeld, Les grandes notions du droit privé, p. 17.

41. Elle a été reconnue comme ayant une valeur constitutionnelle à plusieurs reprises: CC, déc. nº 94/343-344 DC du 27 juillet 1994, Recueil Dalloz, 1995, p. 235 et dernièrement CC, déc. n 2018-768 QPC du 21 mars 2019, Recueil Dalloz, 2019, p. 742. Voir Code civil, art. 16; Charte européenne des droits fondamentaux, art. 1

42. B. Edelman, La personne en danger, Paris, Presses universitaires de France, 1999, p. 508 et 509.

43. X. Lagarde, avant-propos au rapport annuel de la Cour de cassation, Les personnes vulnérables..., p. 65.

44. G. Loiseau, «Des droits humains...»; E. Dreyer, «La dignité opposée à la personne», Recueil Dalloz, 2008, p. 2730.

45. B. Mathieu, «La dignité de la personne humaine: quel droit? quel titulaire?», Recueil Dalloz, 1996, p. 282. Voir, pour le débat, J. Rochfeld, Les grandes notions du droit privé, p. 57.

46. Voir, dans ce même numéro, A. Gosselin-Gorand, L. Fin-Langer, «La vulnérabilité de l'entreprise individuelle».

47. X. Lagarde, avant-propos au rapport annuel de la Cour de cassation, Les personnes vulnérables..., p. 57.

48. Ibid., p. 58 .

49. Voir par exemple: Code pénal, art. 223-15-2 sanctionnant l'abus de faiblesse; Code du travail, art. L. 1132-1. 
ont été donnés en doctrine. Le professeur Xavier Lagarde propose ainsi la définition suivante: «[...] la personne vulnérable est celle qui n'est pas en mesure d'exercer les attributs de la personnalité juridique» ou, en d'autres termes, «celle qui, dans une situation pathologique ou hors norme, [n'est] [...] pas en mesure d'exercer correctement [ses] droits et libertés ${ }^{50}$. Ce critère est sans doute trop large: la vulnérabilité d'une personne ne doit être prise en compte que si l'exposition au risque en raison de sa situation personnelle ou réelle est susceptible de porter atteinte à sa dignité, pouvant exclure la personne de son milieu. Si la personne malade, majeure protégée, mineure ou migrante est objectivement dans une telle situation, dans les autres hypothèses, cela suppose une appréciation subjective, globale et in concreto de la situation, la vulnérabilité étant d'ailleurs susceptible de degrés.

Parmi les vulnérabilités personnelles, outre les aspects liés à la santé physique ou mentale ou à l'âge ${ }^{51}$, il faut sans doute aussi ajouter les faiblesses psychologiques résultant par exemple de l'isolement de la personne. Il pourrait s'agir par exemple du mineur migrant isolé ou de la personne sans papier, y compris en tant que travailleur ${ }^{52}$, ou d'une situation de détresse économique, d'ignorance ou d'inexpérience ${ }^{53}$. La dépendance est une autre cause de vulnérabilité importante et peut s'illustrer notamment dans les nouvelles formes de l'économie numérique ${ }^{54}$.

Parmi les vulnérabilités réelles, résultant du contexte et non de la personne, outre les aspects économiques et sociaux, il faut sans doute ajouter les risques naturels, tels que les inondations visées d'ailleurs par exemple par le Code de l'environnement, les risques sanitaires comme le Covid-19 ou les risques technologiques. En outre, il n'est plus aujourd'hui incongru de parler de vulnérabilité numérique: les personnes dépendent de leur environnement numérique et ne sont plus à l'abri de risques résultant de piratage, de cybercriminalité, de vol d'identité numérique ou d' «illectronisme» qui vont les empêcher d'accéder à leurs droits et de les exercer dans une société de plus en plus digitalisée. Les conditions dans lesquelles est effectué le travail pourraient enfin être facteur de vulnérabilité pour le salarié, voire le travailleur indépendant. Ainsi, une situation de harcèlement ou de travail forcé, voire de servitude, notamment lorsque la personne est elle-même fragile en raison de son âge ou de son état de santé, constituent des facteurs de vulnérabilité pouvant remettre en cause sa dignité. L'ensemble de ces facteurs vont en réalité se combiner et former une chaîne conduisant à des situations non respectueuses de la dignité humaine. Ainsi, des difficultés économiques provoquées par une situation de maladie, de détresse, d'urgence, de besoins, par la conclusion de contrats déséquilibrés peuvent placer la personne dans un isolement social et dans des conditions de logement indignes. Il pourrait s'agir d'une «survulnérabilité», d'une «plurivulnérabilité», ou d'une "vulnérabilité en cascade». L'ensemble de ces risques présente un caractère commun: ils sont irrésistibles, les personnes ne pouvant empêcher leur réalisation. Ce critère, à savoir avoir pour objet ou pour effet de porter atteinte à la dignité de la personne, pourrait préciser les textes qui ne mentionnent pas la forme ou la cause de la vulnérabilité ${ }^{55}$. Comment le droit prend-il alors en compte ces situations?

\section{Le traitement des vulnérabilités par le droit privé}

Certaines mesures sont insuffisantes et d'autres, en revanche, doivent être développées, pour permettre un accompagnement de la personne vulnérable.

\section{A. L'insuffisance des mesures n'assurant pas une prise en charge de la personne vulnérable}

La sanction de l'exploitation de la vulnérabilité et l'utilisation du droit commun pour prévenir ou corriger ses effets sont insuffisantes, car elles ne permettent pas un accompagnement de la personne vulnérable.

\section{L'insuffisance de la sanction de l'exploitation de la vulnérabilité}

Le premier souci du législateur a été de sanctionner les personnes qui exploitent cette vulnérabilité en portant atteinte aux biens de la personne vulnérable ou à sa personne. Le législateur, par une loi no 80-1041 du 23 décembre 1980, a aggravé certaines infractions pénales lorsque la victime était vulnérable ${ }^{56}$. Certaines infractions spécifiques ont également vu le jour, comme le délit d'abus de faiblesse en droit de la consommation ${ }^{57}$ ou celui d'abus de vulnérabilité ${ }^{58}$, qui répriment l'exploitation d'un état d'ignorance ou de faiblesse d'une personne qui n'est pas en mesure d'apprécier la portée de ses engagements ou de déceler les artifices utilisés pour l'inciter à contracter. Si l'objectif

50. X. Lagarde, avant-propos au rapport annuel de la Cour de cassation, Les personnes vulnérables..., p. 59.

51. Cette liste présentée de manière limitative (voir Dictionnaire juridique, C. Puigelier (dir.), «Vulnérabilité») est trop restrictive.

52. La personne sans papier est reconnue comme étant vulnérable par la Cour EDH, 12 mars 2014, Kuric c. Slovénie, $\mathrm{n}^{\circ} 26828 / 06, \S 332$ et 334.

53. Principes du droit européen des contrats, art. 4.109.

54. A. Gosselin-Gorand, L. Fin-Langer, «La vulnérabilité de l'entreprise individuelle».

55. Voir par exemple: Code pénal, art. 225-14 sq. en matière de servitude et de travail forcé; Code du travail, art. L. 8224-2 et L. 8234-1; Code de l'entrée et du séjour des étrangers et du droit d'asile, art. R. 556-12 et R. 213-3.

56. Voir, dans ce même numéro, A. Cerf-Hollender, «Les vulnérabilités nommées et innommées en matière pénale».

57. Code de la consommation, art. L. 121-8.

58. Code pénal, art. 223-15 sq. 
est louable et nécessaire, cette première démarche n'est évidemment pas suffisante.

En effet, les textes relevant du droit pénal sont d'interprétation stricte et ne visent pas toutes les situations. Il est ainsi difficile de comprendre pourquoi certaines infractions, comme le harcèlement moral, font référence aux seules vulnérabilités personnelles alors que d'autres, comme le harcèlement sexuel, font aussi référence aux vulnérabilités réelles. Il est également difficile de comprendre pourquoi les formes les plus graves de harcèlement moral, qui reposent sur une stratégie managériale, ont pu échapper à l'incrimination en raison de la nécessité de prouver le caractère individuel du préjudice ${ }^{59}$. De même, l'abus de faiblesse du Code de la consommation ne protège que l'acheteur, à condition d'être considéré comme un consommateur et sous réserve d'établir l'élément intentionnel ${ }^{60}$. En outre, le nombre réduit des poursuites et la faiblesse des peines prononcées ne dissuadent pas les auteurs de tels faits. Ainsi, la Cour européenne des droits de l'homme a condamné la France pour violation de l'article 4 de la Convention prohibant le travail forcé et la servitude, en raison de l'insuffisance et de l'inefficacité de l'arsenal juridique dans ce domaine, par deux fois ${ }^{61}$. Malgré l'intervention de la loi no $2013-711$ du 5 août 2013 suite à ces condamnations, le nombre des poursuites reste encore trop faible en raison d'une qualification inexacte des faits ${ }^{62}$. Par ailleurs, la personne victime n'est pas prise en charge par ce dispositif, qui ne concerne que l'auteur des faits. Comment aider la victime et réparer son préjudice? Le droit pénal n'apporte aucune solution à ces interrogations.

\section{L'insuffisance de la protection de la personne vulnérable par des dispositions non spécifiques}

La personne vulnérable peut être protégée lors de la conclusion des contrats. Utiliser le droit des contrats spéciaux n'est sans doute pas une approche efficace pour deux raisons essentielles. La première est de considérer nécessairement un consommateur, un preneur ou un salarié comme une personne vulnérable, ce qui n'est pas toujours le cas. Conscient de la difficulté, le législateur a alors mis en place la notion de "particulière vulnérabilité». C'est alors ouvrir la boîte de Pandore et risquer de créer un droit de la consommation ou du travail à plusieurs vitesses, engendrant une rupture d'égalité ${ }^{63}$. Par ailleurs, la réalité peut révéler une vulnérabilité inversée. Ainsi, le bailleur peut parfois être dans une situation de vulnérabilité qu'il est nécessaire de prendre en compte ${ }^{64}$. C'est pourquoi les dispositifs prévus à l'origine dans les contrats spéciaux ont influencé le droit commun des contrats, qui offre ainsi à toute partie au contrat, quelle que soit sa qualité, la possibilité de se prévaloir de ces dispositions protégeant l'équilibre contractuel. La réforme du droit des contrats opérée le 10 février $2016^{65}$ va d'ailleurs dans ce sens. Est ainsi consacrée une obligation pré-contractuelle d'information, pour permettre à la personne de contracter en toute connaissance de cause ${ }^{66}$. De même, sont consacrées la notion de contrat d'adhésion et la lutte contre les clauses abusives, mécanisme d'abord prévu en droit de la consommation puis en droit commercial dans les relations entre distributeur et fournisseur. Cela permet de lutter contre les clauses d'exclusivité ou de non-concurrence, déjà objet de mesure spécifique en droit commercial ou en droit du travail. De même, la violence économique a été introduite dans le Code civil pour lutter contre l'exploitation d'un état de dépendance. C'est d'ailleurs à cette occasion que la notion de personne vulnérable est apparue dans la jurisprudence de la Cour de cassation ${ }^{67}$. La rupture des relations contractuelles est également de plus en plus encadrée pour éviter les résiliations brutales, sans préavis et sans motif. C'est le droit du travail qui, grâce à la mise en place du licenciement justifié par une cause réelle et sérieuse, inspire cette réglementation pour éviter la rupture trop facile d'un contrat indispensable à la dignité de la personne. Cette protection est d'ailleurs renforcée en cas de maladie ou de grossesse. Même les relations entre professionnels, telles que le bail commercial, les relations commerciales bien établies ou celles avec les plateformes numériques d'intermédiation, se rapprochent de ce modèle.

Pour aller plus loin dans cette démarche, il a d'abord été proposé de «bâtir un droit des contractants vulnérables ${ }^{68}$, faisant voler en éclat la distinction entre droit de la consommation, droit de la distribution et droit des contrats spéciaux, voire le droit du travail. Une deuxième piste nous semble préférable, à savoir l'élaboration d'un droit des contrats qualifiés de "life time contracts»" ${ }^{69}$. Cette théorie élaborée au Luxembourg a été construite à partir de

59. Voir, dans ce même numéro, A.-S. Denolle, F. Gabroy, «Vulnérabilité et harcèlement moral: étude comparée du droit de la fonction publique et du droit du travail».

60. Voir le dossier «Le délit d'abus d'ignorance ou de faiblesse», Actualité juridique. Pénal, 2018, p. 219

61. Cour EDH, 26 juillet 2005, Siliadin c. France, $\mathrm{n}^{\circ}$ 73316/o1, Recueil Dalloz, 2006, p. 346, p. 1717; Cour EDH, 11 octobre 2012, C. N. et V. c. France, $\mathrm{n}^{\circ} 67724 / 09$, La semaine juridique, social, $\mathrm{n}^{\circ} 43,23$ octobre 2012, act. 460.

62. Voir, par exemple, B. Lavaud-Legendre, «L'affaire des coiffeurs du boulevard de Strasbourg ou comment le choix de la qualification révèle deux approches d'une même réalité», Revue de droit du travail, 2018, p. 455.

63. Voir D. Bazin-Beust, J.-J. Thouroude, «La vulnérabilité des usagers du service public et des consommateurs».

64. J. Monéger, "De la vulnérabilité en droit des baux", Loyers et copropriété, $\mathrm{n}^{\circ} 5$, mai 2010, repère 5.

65. Ordonnance $\mathrm{n}^{\circ}$ 2016-131 du 10 février 2016 portant réforme du droit des contrats, du régime général et de la preuve des obligations.

66. Code civil., art. 1112-1.

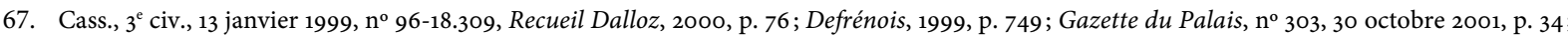
La semaine juridique, édition générale, 1999, I, chron. 143, p. 1076; Revue trimestrielle de droit civil, 1999, p. 382.

68. S. Le Gac-Pech, «Bâtir un droit des contractants vulnérables».

69. D. Hiez, "À propos des life time contracts», Revue trimestrielle de droit civil, 2014, p. 817; Life Time Contracts. Social Longterm Contracts in Labour, Tenancy and Consumer Credit Law, L. Nogler, U. Reifner (dir.), La Haye, Eleven International Publishing, 2014, extraits en ligne: http:// www.eusoco.eu/wp-content/uploads/2013/10/eusoco_book_outline.pdf. 
l'étude du contrat de travail, du crédit à la consommation et du bail d'habitation, qui ont en commun de remplir une fonction particulière, procurer les biens nécessaires aux besoins fondamentaux de la personne. Il s'agit d'éviter que ces contrats soient déséquilibrés, et rompus de manière abusive. Cette proposition de réglementation pourrait être étendue aux contrats nécessaires à un commerçant ou à un entrepreneur individuel ${ }^{70}$.

Le droit civil a également permis d'indemniser les victimes grâce aux règles de la responsabilité civile. La Cour de cassation est à l'origine des évolutions les plus intéressantes pour favoriser l'indemnisation des victimes vulnérables en se fondant sur le principe d'une réparation intégrale. Elle a ainsi étendu la liste des préjudices réparables au-delà du préjudice moral, comme le préjudice d'agrément ${ }^{71}$, sexuel $^{72}$, d'établissement ${ }^{73}$ et même le préjudice permanent exceptionnel indemnisant les préjudices extrapatrimoniaux atypiques, directement liés à un handicap permanent ${ }^{74}$. Cette réparation doit donc tenir compte des spécificités de la personne et notamment de sa vulnérabilité, qui pendant de nombreuses années a été occultée. Il a ainsi fallu attendre 1995 pour que le préjudice subi par les personnes inconscientes soit intégralement réparé ${ }^{75}$.

Deux exemples peuvent être donnés. Tout d'abord, le mécanisme fondé par la loi du 9 avril 1898 et dérogeant au droit commun permet une indemnisation automatique du salarié victime d'un accident du travail, sans qu'il soit nécessaire d'établir une faute de l'employeur. Mais il ne permet pas sa réparation intégrale, en raison du caractère forfaitaire, alors que les victimes de droit commun, si elles doivent établir la faute, peuvent obtenir la réparation de tous leurs préjudices. Pour obtenir la réparation de certains dommages limitativement énumérés ${ }^{76}$, les salariés doivent alors établir la faute inexcusable ${ }^{77}$. Pour tenir compte de cette rupture d'égalité et améliorer la réparation du préjudice subi par les salariés, les juges ont assoupli cette jurisprudence. Depuis une décision du Conseil constitutionnel du 18 juin $2010^{78}$, cette liste n'est plus limitative: d'autres préjudices comme celui sexuel ou d'anxiété peuvent être pris en charge. Au départ cantonné à l'exposition à des poussières d'amiante dans certaines conditions précises ${ }^{79}$, la Cour de cassation a étendu le préjudice d'anxiété à toutes les substances nocives ou toxiques ${ }^{80}$.

De même, la Cour de cassation a opéré un revirement de jurisprudence très récent pour réparer le préjudice subi par des victimes de travail forcé ou en servitude ${ }^{81}$ en faisant droit à une demande d'indemnisation pour le préjudice économique mais aussi moral d'une jeune fille mineure qui a travaillé pour un couple de Marocains sans être rétribuée pendant trois ans. Malgré la condamnation pénale du couple, le juge pénal s'est déclaré incompétent pour accorder cette réparation et le juge civil a rejeté cette demande faute d'établir l'existence d'un contrat de travail. La Cour de cassation casse l'arrêt et indique que «la victime d'une situation de travail forcé ou d'un état de servitude a droit à la réparation intégrale du préjudice tant moral qu'économique ». Elle précise également que le préjudice est aggravé lorsque la victime est mineure, car cette situation de travail forcé ou de servitude compromet son éducation et nuit à son développement physique, mental, spirituel, moral ou social.

Cette réglementation non spécifique de la vulnérabilité doit nécessairement être complétée par d'autres moyens, plus adaptés à la situation des personnes vulnérables, pour préserver ou rétablir leur dignité.

\section{B. La mise en place nécessaire de mesures assurant la prise en charge de la personne vulnérable}

Les techniques individuelles complètent les mesures collectives.

\section{La prise en charge individuelle de la personne vulnérable}

Cette prise en charge individuelle passe par l'accompagnement de la personne. Il peut s'agir de l'attribution, sous certaines conditions, de titres de séjour aux migrants,

70. Idée étudiée par D. Hiez ("À propos des life time contracts») en raison précisément de cette dépendance des petits entrepreneurs individuels. Il énonce le risque d'extension et donc de dénaturation de cette notion et les difficultés de trouver des critères pour définir ces contrats. La taille de l'entreprise ou la forme d'exploitation pourraient être des critères pertinents.

71. Cass., Ass. plén., 19 décembre 2003, La semaine juridique, édition générale, 2004, 10008; Cass., $2^{\mathrm{e}}$ civ., 2 mars $2017, \mathrm{n}^{\circ} 15-27.523$.

72. Cass., $2^{\mathrm{e}}$ civ., 17 juin 2010, Revue trimestrielle de droit civil, 2010, p. 562.

73. Cass., $2^{\mathrm{e}}$ civ., 12 mai 2011, Recueil Dalloz, 2012, p. 47.

74. Cass., $2^{\mathrm{e}}$ civ., 16 janvier 2014, $\mathrm{n}^{\circ} 13-10.566$, Bulletin civil $I I, \mathrm{n}^{\circ}{ }_{13}$, en présence d'un fait dommageable collectif comme une catastrophe naturelle ou un attentat.

75. Cass., $2^{\mathrm{e}}$ civ., 22 février 1995, nº 93-12.644, La semaine juridique, édition générale, 1995, I, 3853; Recueil Dalloz, 1995, somm. 233; Revue trimestrielle de droit civil, 1995, p. 629. À noter que, pour obtenir la réparation du préjudice spécifique de contamination au virus du VIH, il faut toujours la conscience de la personne: Cass., $2^{\mathrm{e}}$ civ., 22 novembre 2012, Revue trimestrielle de droit civil, 2013, p. 123.

76. Code de la Sécurité sociale, art. L. 452-3.

77. Cass. soc., 28 février $2002, \mathrm{n}^{\circ}$ oo-10.051, Bulletin $V, \mathrm{n}^{\circ} 81$

78. CC, déc. no 2010-8 QPC du 18 juin 2010, Revue de droit du travail, 2011, p. 186; Droit social, 2011, p. 1208. La Cour de cassation a opéré un revirement de jurisprudence pour suivre la position du Conseil constitutionnel: Cass., $2^{\mathrm{e}}$ civ., 4 avril 2012, $\mathrm{n}^{\circ}$ 11-14.311, Recueil Dalloz, 2012, p. 1014 et 1098; Droit social, 2012, p. 839.

79. Cass. soc., 3 mars 2015, n 13-20.486, Droit social, 2015, p. 360; La semaine juridique, social, $\mathrm{n}^{\circ} 12,24$ mars 2015, 1106. L'exigence d'une inscription de l'établissement sur une liste administrative n'est désormais plus nécessaire (Cass., Ass. plén., 5 avril 2019, n 18-17.442)

80. Cass. soc., 11 septembre 2019, $\mathrm{n}^{\circ}$ 17-2.4879.

81. Cass. soc., 3 avril 2019, $\mathrm{n}^{\circ}$ 16-20.490, Revue de droit du travail, 2019, p. 529. 
personnes vulnérables en raison d'un déracinement, d'une rupture familiale et des difficultés d'intégration dans le pays d'accueil. Leur situation, si elle est complexe en raison des conséquences politiques et facteur également de vulnérabilité pour certains États, mérite sans doute une plus grande humanité dans le traitement de leur demande. Par ailleurs, le statut de réfugié en date du 28 juillet 1951 devrait être adapté aux nouvelles formes de vulnérabilité émergentes, comme celles résultant des changements climatiques ou des violences faites aux femmes dans leur pays d'origine ${ }^{8_{2}}$. Le Code civil dispose également d'un ensemble de règles spécifiques aux personnes par hypothèse vulnérables, même si ce n'est pas le terme utilisé par le Code civil ${ }^{83}$. Le droit civil est passé d'un droit des incapables à un droit des personnes protégées en 1988, voire à un droit des personnes accompagnées $^{84}$. Il permet non seulement de protéger leur patrimoine en évitant une précarité économique et financière mais aussi leur personne, ainsi que leurs droits extrapatrimoniaux voire fondamentaux ${ }^{85}$ en les aidant à garder leur autonomie et leur dignité malgré leurs difficultés physiques ou mentales. Elles sont prises en fonction d'une appréciation in concreto de l'altération réelle de leurs facultés mentales. Il faut cependant préciser que toutes les personnes vulnérables n'ont pas nécessairement besoin de telles mesures de protection civile, notamment en cas de vulnérabilité économique et/ou sociale.

Certaines mesures de prévention visent à limiter les risques financiers, comme les mécanismes mettant à l'abri le patrimoine personnel de l'entrepreneur individuel, par le biais par exemple de l'insaisissabilité de son logement ou la séparation de son patrimoine grâce à l'EIRL (entreprise individuelle à responsabilité limitée) en deux sans création d'une personne morale. S'il s'agit de protéger le logement indispensable à la protection de la dignité de la personne ${ }^{86}$, il n'est pas certain que ces dispositifs soient les plus efficaces, car ils peuvent constituer une nouvelle cause de vulnérabilité: ils vont avoir pour effet de limiter le gage des créanciers et donc les garanties financières nécessaires à l'exercice d'une activité économique. Ils créent alors l'illusion d'une irresponsabilité et d'une protection qui peut voler en éclat. Les créanciers vont ainsi demander un cautionnement au conjoint marié sous le régime de la séparation de biens ou des taux d'intérêt plus élevés en raison du risque financier. D'autres mesures visent à aménager les dettes, voire à les effacer totalement, dans le cadre d'un rétablissement personnel étendu aux entrepreneurs individuels grâce à la procédure de rétablissement professionnel ${ }^{87}$. Ces mécanismes sont inspirés d'une logique sociale de solidarité ${ }^{88}$ et traduisent cette exigence de dignité ${ }^{89}$, car ils visent à éviter l'exclusion sociale ${ }^{90}$. Cela permet une protection contre les «accidents de la vie». Le surendettement bénéficie aussi aux personnes qui ont accumulé du crédit de manière parfois excessive mais en raison d'une imprévoyance, d'une inconséquence. Ne sont exclues que les personnes qui ont organisé de manière délibérée leur endettement excessif, la bonne foi n'étant plus caractérisée. L'organisation d'une insolvabilité peut même constituer une infraction pénale ${ }^{91}$. La même exigence apparaît dans le rétablissement professionnel, puisqu'en présence d'une mauvaise foi, sera ouverte une liquidation judiciaire. L'inconvénient de ce dispositif réside dans les risques de surprotection parfois du surendetté présumé être vulnérable, sacrifiant alors les droits des créanciers qui peuvent aussi être à leur tour vulnérables ${ }^{92}$. Il est sans doute préférable d'utiliser d'autres modes de prise en charge collective pour tenir compte de cette vulnérabilité économique et sociale, la prise en charge ne reposant alors plus sur les cocontractants.

\section{La prise en charge collective de la personne vulnérable}

Le fondement de la lutte contre les exclusions repose sur la dignité humaine et est clairement affirmé notamment dans l'article L. 115-1 du Code de l'action sociale et des familles. Le droit de la protection sociale apporte un soutien le plus souvent financier aux personnes les plus vulnérables en présence de risques sociaux au nom de la solidarité nationale ${ }^{93}$, en complément des mécanismes

82. Voir, dans ce même numéro, C.-A. Chassin, A. Korsakoff, L. Mauger-Vielpeau, «La vulnérabilité des migrants». Lors de la journée d'étude ayant donné lieu à cet article (journée d'étude intitulée «Vulnérabilités et droit, regards croisés», qui s'est tenue le 7 février 2020 à l'université de Caen Normandie, sous la direction de Mathias Couturier, Delphine Bazin-Beust, Samuel Etoa, Aurore Catherine et Laurence Fin-Langer), A. Korsakoff a démontré que ce statut n'était pas nécessairement adapté aux femmes persécutées dans leur pays, malgré les évolutions récentes du droit.

83. Cour de cassation, Les personnes vulnérables..., p. 97. L'expression est parfois utilisée par les juges du fond: Cass., $1^{\text {re }}$ civ., 7 juin 2006 , $\mathrm{n}^{\circ} \mathrm{04}-10.125$ Cass., $1^{\text {re }}$ civ., 12 décembre 2006, $n^{\circ}$ 05-21.312.

84. P. Malaurie, avant-propos à Droit des personnes: la protection des mineurs et des majeurs, $9^{\mathrm{e}}$ éd., Issy-les-Moulineaux, LGDJ, 2017.

85. A. Batteur, Droit des personnes, $\mathrm{n}^{\circ} 1270$ sq. : la loi $\mathrm{n}^{\circ} 68-5$ du 3 janvier 1968 portant réforme du droit des incapables majeurs n'organisait qu'une protection des biens, étendue à leur personne par la Cour de cassation (Cass., $1^{\text {re }}$ civ., 18 avril 1989, $\mathrm{n}^{\circ} 87-14563$ ), extension consacrée par la loi $\mathrm{n}^{\circ}$ 2007-308 du 5 mars 2007 (Code civil, art. 415). La loi n 2019-222 de programmation 2018-2022 et de réforme pour la justice du 23 mars 2019 protège également l'exercice de certains droits fondamentaux comme en matière de mariage et de droit de vote.

86. CC, déc. nº 94-359 DC du 19 janvier 1985. Voir aussi Logement et vulnérabilité, D. Guérin, F.-X. Roux-Demare (dir.), Bayonne - Issy-les-Moulineaux, Institut universitaire Varenne - LGDJ (Colloques et essais), 2016.

87. Les conditions d'ouverture sont assez strictes: absence de bien immobilier, absence de salariés ou de contentieux social, un actif d'une valeur inférieure à 5000 euros. Voir A. Gosselin-Gorand, L. Fin-Langer, «La vulnérabilité de l'entreprise individuelle».

88. Cour de cassation, Les personnes vulnérables, p. 129.

89. J.-B. Racine, «Faut-il encore payer ses dettes? Le cas du surendettement des particuliers», Les petites affiches, n० 63, 29 mars 2006, p. 37.

90. Voir V. Martineau-Bourgninaud, Procédures de surendettement des particuliers et rétablissement personnel, Issy-les-Moulineaux, LGDJ, 2018, ${ }^{\circ} 27$ sq.

91. Ibid., $\mathrm{n}^{\circ} 211$ sq.

92. Ibid., $\mathrm{n}^{\circ} 40$

93. P. Morvan, Droit de la protection sociale, $8^{\mathrm{e}}$ éd., Paris, LexisNexis, 2017, $\mathrm{n}^{\mathrm{o}} 1$. 
d'épargne individuelle parfois impossibles et des assurances facultatives privées ${ }^{94}$. Depuis sa mise en place en 1945, un débat existe pour savoir si ces prestations doivent avoir un caractère universel, et être accordées à tout individu, ou si elles sont liées à un statut particulier, comme l'exercice d'une activité professionnelle ${ }^{95}$. Si le Conseil national de la résistance voulait donner à tout citoyen des moyens d'existence à chaque fois qu'il est incapable de se les procurer par son travail, l'ordonnance du 4 octobre 1945 a limité cette protection aux seuls travailleurs, entendus comme salariés ${ }^{96}$. Il faut cependant signaler une évolution vers une universalisation du régime de protection et un alignement progressif, mais encore insuffisant ou incomplet, sur le régime des salariés, lorsque l'entrepreneur individuel se trouve dans l'impossibilité temporaire de fournir sa prestation et donc de générer un revenu. Ainsi, les entrepreneuses peuvent bénéficier d'un congé maternité proche de celui des salariées et désormais tous peuvent toucher une allocation de fin d'activité, mais à des conditions très restrictives ${ }^{97}$. De même, les mesures prises en raison du Covid-19 visent tous les travailleurs, selon des modalités analogues. Reste une différence notable avec les salariés: il n'y a aucune prise en charge des accidents du travail ou de maladies professionnelles bien que les entrepreneurs soient fortement exposés à ces risques. C'est aujourd'hui une question importante pour les travailleurs utilisant les plateformes de mise en relation numérique: s'ils ont l'apparence de l'indépendance, ils sont en réalité sous leur subordination économique et leur surveillance numérique ${ }^{98}$. Ils prennent parfois des risques inconsidérés mettant en danger leur santé et leur sécurité, vulnérabilité qui a d'ailleurs justifié la mise en place d'un régime particulier dans la loi $n^{\circ}$ 2019/1428 du 24 décembre 2019 d'orientation des mobilités pour certaines plateformes. Pour bénéficier d'une protection dont ils sont privés en raison de leur statut d'indépendant, ces travailleurs agissent en justice pour obtenir la requalification de leur relation en contrat de travail, demande que les tribunaux acceptent désormais ${ }^{99}$

À côté de la Sécurité sociale, reposant sur une idée d'assurance collective obligatoire et contributive, existe également les mécanismes de l'aide sociale fondés sur l'idée d'assistance sociale, de charité non contributive mais liée à des conditions de ressources ${ }^{100}$. L'aide sociale vise alors à garantir, au-delà de la protection de la personne vulnérable, la dignité humaine par un accompagnement financier mais aussi social ${ }^{101}$. Le RSA (revenu de solidarité active) et la prime d'activité en cas d'activité professionnelle relèvent de cette logique: peut en bénéficier toute personne de plus de 25 ans y compris l'entrepreneur individuel qui ne dispose pas d'un revenu minimum pour éviter l'exclusion sociale ${ }^{102}$. Le gouvernement a ainsi annoncé de nombreuses mesures au cours du mois de mars 2020 pour éviter que la crise du Covid-19 soit un facteur supplémentaire de vulnérabilité pour toutes les personnes. Ces mécanismes reposant sur la solidarité nationale complètent utilement les mesures civiles de protection. Toute la difficulté est de trouver un équilibre pour préserver les différents intérêts et pour éviter une surprotection tout en accompagnant la personne en fonction de ses besoins concrets et de son autonomie.

96. M. Borgetto, «La Sécurité sociale à l'épreuve du principe d'universalité», Revue de droit sanitaire et social, 2016, p. 11. Cette différence de régime entre les salariés et les autres travailleurs a été jugée non discriminatoire et conforme à la Convention européenne de sauvegarde des droits de l'homme: Cass. soc., 11 mai 2001, n 99-20.420, Droit social, 2001, p. 778.

97. Il s'agit de l'allocation des travailleurs indépendants (Code du travail, art. L. 5424-25). Voir Feuillet Rapide Social Francis Lefebvre, $\mathrm{n}^{\circ} 23,2019$, ${ }^{\circ}{ }^{\circ}$. 98. I. Desbarats, «Quel statut social pour les travailleurs des plateformes numériques? La RSE en renfort de la loi », Droit social, 2017, p. 971.

99. Cass., $2^{\text {e }}$ civ., 28 novembre 2019, $\mathrm{n}^{\circ}$ 18-15.333 et 18-15.348; Cass. soc., 4 mars 2020, $\mathrm{n}^{\circ}$ 19-13.316, arrêt Uber.

100. Code de l'action sociale et des familles, art. L. 115-1.

101. P. Morvan, Droit de la protection sociale, $\mathrm{n}^{\circ} 473$

102. Code de l'action sociale et des familles, art. L. 262-1 et L. 115-2. 